TEMAS DE ACTUALIDAD Rev Chil Salud Pública 2014; Vol 18 (1): 19-24

\section{REFORMAS AL SISTEMA CHILENO DE SEGURIDAD Y SALUD EN EL TRABAJO (SST) ${ }^{1}$ \\ Reform of the Chilean System for Occupational Safety and Health}

\section{RESUMEN}

Luego del suceso "Rescate en la Mina San José" se abrió un debate sobre la necesidad de hacer modificaciones al sistema de protección de seguridad $y$ salud en el trabajo chileno, se crearon comisiones, se hicieron informes $y$ propuestas y se presentaron varios proyectos de ley.

En el presente artículo se analiza el escenario actual, los avances y situaciones pendientes a tres años del accidente.

\section{ABSTRACT}

After the San Jose Mine Rescue incident, a debate was opened in the country over the need to modify the present occupational safety and health system in Chile. Several commissions were formed and many reports and proposals were made. Also some legislation projects were sent to Congress for its approval.

This article analyses the current scenery, advances and pending issues on these matter after three years of the San Jose Mine successful rescue.

\section{INTRODUCCIÓN}

El 5 de agosto de 2010 se produjo un derrumbe en la mina de cobre San José, ubicada cerca de Copiapó, en la región de Atacama, que dejó 33
1. Trabajo de la autora publicado en: http://www.saludpublica.uchile.cl/home/images/pdf/ ARTiCULO.pdf 
mineros atrapados durante 70 días, a 700 metros de profundidad.

El exitoso rescate implicó un gran despliegue técnico y concitó la atención nacional y mundial. ${ }^{2} \mathrm{Su}$ costo total fue de 20 millones de dólares, ${ }^{3}$ cubierto por la empresa estatal Corporación del Cobre de Chile, Codelco, en un 75 por ciento. ${ }^{4}$

En noviembre de 2010, el Consejo de Defensa del Estado demandó a la minera San Esteban, propietaria de la Mina San José, exigiendo el reembolso de lo gastado, cifra estimada inicialmente en US\$ 22 millones. ${ }^{3}$ Finalmente la demanda se presentó por US\$

2. Entre las 8:15 y 9 de la noche, Fox News atrajo a una audiencia de 7,1 millones de espectadores. 2 millones 700 mil personas siguieron el acontecimiento desde CNN. La cifra más alta desde el día de las elecciones en Estados Unidos, en 2008. Más de 6,8 millones de personas presenciaron la salida del primer minero desde BBC News, informó The Guardian.

3. http://actualidad.orange.es/sociedad/costo-del-rescate-33-mineros-en-chile-asciende-20-millones-dolares.htm

4. Ocho millones de dólares, corresponden a los contratos de las tres máquinas que perforaron la mina San José para llegar hasta el sitio donde los obreros estaban guarecidos. La Strata 950, del "Plan A", tuvo un costo total aproximado de 3 millones de dólares, mientras que la RIG-421, la perforadora petrolera del "plan C", fue la más cara, con un costo de unos 3,5 millones de dólares. La perforadora Schramm T-130, que trabajó en el "plan B” y es la que consiguió dar con los mineros, tuvo un costo de 1,1 millones de dólares. Esta cifra no incluye la contratación de la máquina, que fue cedida por la Compañía Minera Collahuasi, sino la importación de los martillos y los bienes y servicios asociados a su uso. Codelco incluyó entre los gastos el costo de las operaciones de sondaje, el izado de la cápsula de rescate, mediciones, movimientos de tierra, trabajos de ingeniería, transporte y otras partidas, que suman 7,4 millones de dólares. La Empresa Nacional del Petróleo, Enap, la petrolera estatal, gastó cerca de un millón de dólares por el combustible utilizado en las operaciones. La Intendencia y la Gobernación de la región de Atacama y la Oficina Nacional de Emergencia (Onemi) también gastaron un millón de dólares en el traslado de las familias, pasajes aéreos para los socorristas, alimentación y otros elementos. La Armada destinó 34 millones de pesos (unos 69 mil dólares) en la fabricación de tres cápsulas "Fénix" para sacar a los mineros desde el interior del yacimiento. Las Fuerzas Armadas gastaron alrededor de medio millón de dólares, principalmente por la utilización de helicópteros de la Fuerza Aérea de Chile (FACh).
10,3 millones, al descontarse los gastos de las empresas que colaboraron con Codelco.

En marzo de 2012, luego de un acuerdo entre el síndico de quiebras y el Consejo de Defensa del Estado, la minera San Esteban se comprometió a pagar al Fisco de Chile la suma de 5 millones de dólares, que se obtendrán de la venta de los activos mineros de la compañía.

Por otra parte, el 1 de agosto de 2013, la Fiscalía de Atacama cerró la investigación criminal, sin acusados, por cuanto "no había convicción para formular una acusación" contra los dueños de la minera San Esteban.

\section{DIAGNÓSTICO DEL SISTEMA CHILENO}

El suceso Mina San José colocó por breve tiempo en la agenda pública el tema de la seguridad y salud en el trabajo, permitiendo a los trabajadores y expertos manifestar la necesidad de hacer transformaciones al Sistema de Protección.

Chile cuenta con un Seguro contra Accidentes del Trabajo y Enfermedades Profesionales (Ley 16.744), que ha permitido en los últimos 45 años mejorar las condiciones de trabajo en materia de seguridad y salud $y$, principalmente, las prestaciones reparatorias en caso de accidentes o enfermedades profesionales. Sin embargo, aunque las cifras demuestran una baja en la tasa de accidentabilidad, la tasa de fatalidad, sobre todo en minería, es alta, muy por encima de los países desarrollados. Respecto de la incidencia y prevalencia de enfermedades profesionales, no hay información.

El sistema actual es insuficiente y se requiere contar con un sistema de protección que ponga el énfasis en lo preventivo y no en lo curativo. La gran mayoría de los países cuenta con una ley de prevención -que en Chile no existe- y con políticas públicas destinadas a crear una "cultura de la prevención" en todos los niveles.

El diagnóstico compartido es que el sistema de fiscalización presenta grandes debilidades, como ha sido representado por estudios 
de la Universidad Católica, ${ }^{5}$ organizaciones de trabajadores ${ }^{6}$ y la Comisión Asesora Presidencial para la Seguridad en el Trabajo. ${ }^{7}$

La existencia de multiplicidad de organismos genera confusión, duplicidad de esfuerzos, mala utilización de los recursos, contiendas de competencia entre ellos y pro-

5. Brahm F., Singer M., Valenzuela L., Ramírez C.; Facultad de Ciencias Económicas y Administrativas de la Pontificia Universidad Católica y la OIT. "Comparación internacional de sistemas de salud y seguridad laboral informe final", p. 10. "En cuanto al rol del estado en la SSL, Chile muestra un déficit de fiscalizaciones y número de fiscalizadores. Para alcanzar el estándar de la OCDE habría que aumentar las fiscalizaciones en un $40 \%$ y aumentar el número de fiscalizadores al doble...”. Nov. 2010.

6. Informe Final Comisión CUT Seguridad y Salud de los Trabajadores, 14 de noviembre 2010, p. 11. "La fiscalización de la Dirección del Trabajo está fuertemente orientada hacia los aspectos laborales de las relaciones de trabajo. Se trata de inspectores polivalentes cuya alta experticia en materias laborales, no va acompañada de una especialización en los riesgos específicos para la salud y la seguridad. Es evidente que aspectos laborales como las largas jornadas de trabajo y los descansos, bien inspeccionados por la Dirección del Trabajo, son muy importantes en riesgos del trabajo. Sin embargo, la fiscalización se centra en el chequeo documental de aspectos más bien burocráticos (reglamentos internos, actas de comités paritarios) o en condiciones de seguridad e higiene básicas (condiciones de comedores y baños), sin entrar a la identificación de los peligros materiales y riesgos para la salud que están en la base de los accidentes y enfermedades. Cabe señalar que, a pesar de ello, se detectan problemas”.

7. Comisión Asesora Presidencial para la Seguridad en el Trabajo. Informe Final. Noviembre 2010, p. 76. "Se trata de una institucionalidad esencialmente dispersa y de una diversidad de órganos públicos con competencias y atribuciones en la materia, a veces parceladas, y en otras superpuestas. Esta situación da origen a problemas de coordinación que no están bien resueltos. En particular, los criterios usados para definir responsabilidades entre las entidades de fiscalización no son los adecuados; no existen objetivos ni metas comunes; la comunicación entre entidades que fiscalizan, y entre éstas y las entidades administradoras del seguro, no es siempre adecuada y oportuna; y coexisten diferentes procedimientos, criterios, multas, y sistema sancionatorio en cada institución. Destaca la falta de políticas integradas y la ausencia de estrategias de fiscalización que se ajusten a la realidad del mercado. Al mismo tiempo, y en parte como consecuencia de la falta de coordinación, la calidad, cobertura e impacto de la fiscalización no es la más adecuada. Además, en la mayoría de los casos las fiscalizaciones tienen carácter reactivo". blemas de planificación, provocando en ocasiones dobles y triples fiscalizaciones $\mathrm{y}$, en otras, ausencia de la misma.

Adicionalmente, todas las entidades carecen de facultades y recursos suficientes para cumplir sus cometidos en forma eficiente y con calidad.

La normativa está disgregada y desactualizada, hay subregistro de siniestralidad, sobre todo de enfermedades profesionales, escasa información estadística, insuficiente y desprolija fiscalización, y cobertura parcial del seguro.

El trabajo y las relaciones laborales actualmente se desenvuelven en un marco de baja participación sindical y negociación colectiva, gran disparidad de salarios, jornadas de trabajo extensas, alta rotación o movilidad laboral, condiciones extremas de trabajo y presiones por mayor productividad y menores costos.

En este contexto, el deber de protección y la conducta preventiva requieren de una decidida y permanente promoción, regulación y supervigilancia por parte del Estado.

A la fecha los avances son insuficientes, $y$ pese a la aprobación del Convenio 187 de la OIT, ${ }^{8}$ el país aún no cuenta con una Política de Seguridad y Salud en el Trabajo.

\section{INICIATIVAS LEGALES}

Aprobadas:

1. Comité de Ministros para la Seguridad y Salud en el Trabajo, Decreto 20/2011 del Ministerio del Trabajo y Previsión Social. El Comité inició su trabajo el día 6 de enero del año 2012, presidido por el Ministro del Trabajo y Previsión Social e integrado, además, por los ministros de Defensa Nacional; de Economía, Fomento y Turismo; de Salud; de Agricultura; de

8. El Convenio 187 presenta un marco promocional para el tratamiento sistemático y coherente de la seguridad y salud en el trabajo y promueve el reconocimiento y la aplicación de otros convenios de la OIT sobre seguridad y salud. El Convenio 187 fue adoptado durante la

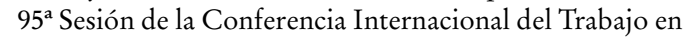
junio de 2006. 
Minería; y de Transportes y Telecomunicaciones. Sus funciones son: asesorar, elaborar la política, velar por la aplicación de normas y estándares coherentes con la política, y armónicas entre distintas entidades y organismos con competencias para dictarlas.

2. Consejo Consultivo para la Seguridad y Salud en el Trabajo, Decretos 19 y 33 (2011) del Ministerio del Trabajo y Previsión Social. Inició sus funciones el día 15 de marzo de 2012. Lo integran cinco miembros, nombrados por el Presidente de la República, uno de los cuales deberá pertenecer o haber pertenecido a organizaciones de empleadores; otro deberá pertenecer o haber pertenecido a organizaciones de trabajadores, contando ambos con experiencia en la implementación de la normativa sobre seguridad y salud en el trabajo, o en materia laboral o de previsión social, o en su promoción y protección. Los restantes tres consejeros deberán ser profesionales de reconocida experiencia y trayectoria en el ámbito de la seguridad y salud en el trabajo, o en materia laboral o de previsión social, uno de los cuales asumirá como presidente del Consejo, a elección del Presidente de la República, y mientras cuente con su confianza. La función de este Consejo es analizar y emitir informes con sus opiniones.

3. Ratificación del Convenio OIT 187, Marco Promocional para la Seguridad y Salud en el Trabajo a través del Decreto 72 de 2011 del Ministerio de Relaciones Exteriores. Ésta debe ser una política nacional emitida con representatividad de las organizaciones sociales, con objetivos, metas, plazos, prioridades y recursos. Debe contar además con una infraestructura legal e institucional para la aplicación de la política y los programas. Así también, debe contar con definiciones muy claras de los derechos, deberes y responsabilidades de todos los actores y favorecer una 'cultura de prevención', el derecho a un medio ambiente de trabajo sano y seguro que se respeta en todos los niveles.

\section{RECHAZADA}

1. Creación de una Superintendencia de Minería (Boletín 7848-08). Esta superintendencia fue rechazada por los parlamentarios de la Concertación con el fundamento que de aprobarse perjudicaría a la pequeña minería con multas de hasta 10.000 UTM, y ello debe legislarse en forma separada. Por otra parte se estimó que Sernageomin no tiene recursos suficientes para fiscalizar, que este proyecto elimina Cochilco y además no se consideró la opinión de los trabajadores.

\section{INICIATIVAS PENDIENTES}

1. Ley Orgánica de la Superintendencia de Seguridad Social (Boletín 7829-13) 1 de agosto de 2011. Está en tercer trámite constitucional en la Cámara de Diputados. Sus cargos directivos son de Alta Dirección Pública y de acuerdo a normas incorporadas por el Senado, la constitución de sociedades y organismos filiales de las instituciones de previsión social sometidas a la vigilancia integral de la Superintendencia de Seguridad Social (Suseso), deberá ser autorizada por ese organismo. Se incrementan las multas de 50 a 100 unidades de fomento, las que serán aplicables a los consejeros, directores, vicepresidentes y administradores de las instituciones fiscalizadas que no presten declaración en los casos que sean requeridos. Se agrega que las sanciones administrativas se aplicarán sin perjuicio de las responsabilidades penales. Las funciones de esta Superintendencia son:

- Sistematizar y proponer la estandarización de la normativa sobre seguridad y salud en el trabajo, propendiendo a su uniformidad y su revisión periódica.

- Administrar y mantener el Sistema Nacional de Información de Seguridad y Salud en el Trabajo, Sisesat.

- Elaborar la Memoria Anual del Sistema Nacional de Seguridad y Salud Laboral, que incluirá los resultados alcanzados, los 
principales hitos en el desarrollo de la Política Nacional de Seguridad y Salud en el Trabajo, y los avances que se registren en el logro de los objetivos, indicando niveles de cumplimiento de los mismos y perspectivas para el futuro.

- Recopilar, consolidar y sistematizar la información que proporcionen los organismos administradores de la Ley 16.744 y las diversas instituciones públicas con competencias en materias de seguridad y salud laboral, directamente o a través del Sisesat.

- Cargos Alta Dirección Pública. Normas incorporadas por el Senado:

- Acerca del personal directivo, se aumenta de 7 a 10 por ciento el personal a contrata de esa Superintendencia, al que se le pueden encomendar funciones de carácter directivo.

2. Ley Orgánica de Mutualidades (Boletín 8573-13) 6 de septiembre de 2012. Está en segundo trámite constitucional en el Senado. Fundamentos y principales cambios propuestos:

- 44 años sin modificaciones.

- 80,25 por ciento de los trabajadores afiliados está en una mutualidad.

- Búsqueda de equilibrio entre sus objetivos económicos y sociales.

- Buenas prácticas de los gobiernos corporativos.

- Garantizar el destino de los recursos para los fines propios.

- Resguardar la entrega de las prestaciones.

- Regular conflictos de intereses.

- Asegurar transparencia de la información.

- Regular uso de información reservada.

- Regular el rol de las Juntas de Adherentes.

- Inversiones de Fondos de Reservas.

- Fusiones, intervención y disolución.

- Solo podrán rechazar una solicitud de adhesión o afiliación por razones de insuficiente infraestructura para entregar las prestaciones que obliga la ley en la localidad donde se desempeñen los trabajadores respectivos, previa autorización expresa de la Superintendencia de Seguridad Social.
- Se regula también las causales para excluir de una Mutualidad a una entidad empleadora adherente o a un trabajador independiente afiliado. En particular, para tomar esta decisión el Directorio de las mutualidades solo podrá invocar el incumplimiento grave y reiterado de las medidas de prevención de riesgos y el atraso por más de dos meses en el pago de las cotizaciones de la ley 16.744 .

3. Reforma a la Ley 16.744 (Boletín 8971-13) 4 de junio de 2013. Pasa a Comisión de Trabajo y Seguridad Social y a Comisión de Hacienda.

\section{PRINCIPALES CAMBIOS PROPUESTOS}

- Separa binomio (Instituto de Seguridad Laboral (ISL) y Servicios de Salud).

- Aclara obligaciones, organismos, empleadores y trabajadores.

- Fiscalización de empresas: Concentra facultades en la Dirección del Trabajo (Dirtrab) con suspensión inmediata de labores.

- Fiscalización de entidades públicas: en las Autoridades Sanitarias.

- Evaluación de incapacidades en cada organismo administrador.

- Investigación de accidentes.

- Autoevaluación de las empresas.

- Sistema de Gestión de Seguridad y Salud en el Trabajo (SGSST) en el caso de más de 50 trabajadores.

- El DPR puede externalizar funciones.

- Si hay agentes "podrán" incorporar a programa de vigilancia.

\section{PROPUESTAS DE LA ESCUELA}

La SST forma parte esencial del Programa de Salud Ocupacional de la Escuela de Salud Publica para el período, con énfasis en investigación, docencia y relaciones con el entorno.

En el escenario descrito, nuestro trabajo no puede limitarse al ámbito médico o meramente técnico; es necesario hacer un ejer- 
cicio de análisis permanente del impacto de estas variables y consensuar opiniones entre los académicos, que permitan levantar una propuesta y una agenda de trabajo con los actores sociales y políticos, erigiéndose en un actor relevante y ejercer el liderazgo en el tema.

Por ello, dado que Chile no cuenta con una ley específica en materia de seguridad y salud en el trabajo, ni políticas destinadas a crear una "Cultura de la Prevención", la Escuela está desarrollando un proyecto que propone una ley, que considera las siguientes finalidades:

1. Establecer los principios, bases e ideas matrices del Sistema Único de Seguridad y Salud en el Trabajo, que tendría por finalidad la protección a la vida, la salud y la integridad física y psíquica de los trabajadores y la creación de una "Cultura de Prevención", cuyo marco regulatorio estaría conformado por la Ley del Seguro Social de Seguridad y Salud en el Trabajo y por la Ley de Seguridad y Salud en el Trabajo, y la normativa técnica y reglamentaria que las complemente.

2. Establecer la institucionalidad, obligaciones y roles de los organismos del Estado para cumplir con su función promotora, preventiva, reguladora, fiscalizadora y de resolución de conflictos en esta materia.

3. Establecer un procedimiento normativo único, obligatorio y participativo para las actualizaciones y nuevas regulaciones.

4. Establecer los derechos, obligaciones, roles y funciones de empleadores, trabajadores y órganos de participación.

5. Definir los procesos productivos que se considerarían peligrosos y se someterían a una gestión, regulación y fiscalización especiales.

6. Definir los instrumentos de promoción y prevención de riesgos, obligatorios según tipo de faena o proceso productivo.

7. Establecer estándares obligatorios de seguridad y salud ante peligros, agentes y condiciones presentes en los procesos productivos y sitios de trabajo y para ciertos trabajadores.
Se hace necesario imprimir un ritmo más acelerado a las reformas, con participación activa de todos los actores y expertos en el tema, a fin de incorporar cambios necesarios que hasta ahora están ausentes, y encontrar la forma de colocar el tema en la agenda electoral. 